\title{
Premiers résultats d'une étude géophysique sur le flanc nord des glissements de Storegga (Norvège)
}

\section{First results of a geophysical survey on the northern flank of the Storegga slides (Norway).}

Hervé Nouzé ${ }^{a}{ }^{*}$, Isabelle Contrucci ${ }^{a}$, Jean-Paul Foucher ${ }^{a}$, Bruno Marsset ${ }^{a}$, Yannick Thomas ${ }^{a}$, Estelle Thereau ${ }^{\mathrm{a}}$, Alain Normand ${ }^{\mathrm{a}}$, Éliane Le Drezen ${ }^{\mathrm{a}}$, Stéphane Didailler ${ }^{\mathrm{a}}$, Jean-Pierre Regnault ${ }^{\mathrm{a}}$, Sandie Le Conte $^{\mathrm{a}}$, Stéphanie Guidart ${ }^{\mathrm{b}}$, Wim Lekens ${ }^{\mathrm{c}}$, Simon Dean ${ }^{\mathrm{d}}$ and Alexandre Throo ${ }^{\mathrm{e}}$

\author{
aIfremer, Centre de Brest, DRO/GM, BP 70, 29280 Plouzané, France \\ ${ }^{\mathrm{b}}$ Department of Geology, University of Tromsoe, Norway \\ ${ }^{\mathrm{C}}$ Geologisk Institut, Bergen, Norway \\ ${ }^{\mathrm{d}}$ Southampton Oceanography Centre, Southampton, UK \\ Institut français du pétrole, 1, av. de Bois-Préau, 92852 Rueil Malmaison cedex, France \\ *: Corresponding author : Hervé Nouzé, Ifremer, Centre de Brest, DRO/GM, BP 70, 29280 Plouzané, \\ France Tél. : (33) (0)2 98224709 / Fax (33) (0)2 98224549 Herve.Nouze@ifremer.fr
}

\begin{abstract}
The Storegga slides, off Norway, are among the largest submarine slides ever known on a continental slope. The HYDRATECH cruise on N/O Le Suroît aimed at a high-resolution survey of an area at the northern boundary of the slides. This survey images in great detail the bottom simulating reflector (BSR) extent and properties, the various fluid escape structures and the sediment deformations. The combination and the quality of the data help to understand the still poorly constrained relationships between fluid escapes, gas hydrates and slope stability in the survey area. To cite this article: H. Nouzé et al., C. R. Geoscience 336 (2004).
\end{abstract}

Résumé : Les glissements de Storegga, au large de la Norvège, sont les plus grands glissements connus sur une pente continentale. Lors de la campagne Hydratech sur le N/O Le Suroît, un levé géophysique à haute résolution d'un secteur du flanc nord des glissements a été effectué. Ce levé permet d'imager en détail l'extension et les propriétés du Bottom Simulating Reflector (BSR), les structures d'échappement de fluides et les déformations sédimentaires. La combinaison des différentes données acquises ainsi que leur qualité permettent de mieux comprendre les interactions encore mal contraintes entre fluides, hydrates de gaz et glissements sur la zone d'étude. Pour citer cet article : H. Nouzé et al., C. R. Geoscience 336 (2004).

Mots-clés: Storegga; hydrates de gaz; sorties de fluides; stabilité des pentes Keywords: Storegga; gas hydrates; fluid escapes; slope stability 


\section{Abridged English version}

The end of the Norwegian margin formation during the opening of the north Atlantic ocean coincides with the Paleocene-Eocene transition about 55 Ma ago [6]. From the Miocene times to the Pliocene times, the fine grained hemipelagic sediments of the Kai formation are deposited on the margin. The Kai formation is affected by a polygonal fault system in relation with fluid expulsion [1]. From the Pliocene times to the Pleistocene times, the thick prism of sediments of the Naust formation is deposited on the shelf during several glacial episodes [14]. From 2.5 to $1.3 \mathrm{Ma}$, the prograding platform fills the Storegga depression. During the last 1.3 Ma, the sedimentation is controlled by glacial and interglacial cycles [11], with a maximum activity at 0.6-0.5 Ma when the Fenno-scandian ice sheet repeatedly covers the area. The Storegga slides have been mapped over more than $800 \mathrm{~km}$, from the head wall, on the top of the continental slope at about $400 \mathrm{~m}$ water depth down to the Norwegian basin abyssal plain at $3600 \mathrm{~m}$ water depth. [4]. Eight retrogressive slides, triggered over a short time period have been identified [8]. Whereas the main phase of the Storegga slides was dated at about 7250-7300 years C14 (8150-8200 bp), recent studies on the northern flank of the slides show evidence for more recent sliding episodes: $5000+/-300$ years C14 et 22002500 years C14. Gas hydrates are composed of gas molecules trapped within water molecules cages [12]. Gas hydrates formation is mainly controlled by thermo dynamical conditions (high pressure and low temperature) that occur on most of the continental margins at water depths between 500 and $1000 \mathrm{~m}$. The occurrence of marine gas hydrates in the sediments is mainly inferred from the observation on seismic sections of a Bottom Simulating Reflector (BSR). Geophysical studies [9] have shown that the BSR is generated at the transition between sediments containing a variable amount of solid gas hydrate above and sediments containing a small volumetric fraction of free gas below. The stability field for gas hydrate being mainly P-T dependant, and isotherms approximately parallel to the sea floor, the BSR parallels the seabed, shows a polarity opposite to the seafloor polarity and often cuts across sedimentary layer reflections. In the Storegga area, BSRs have been identified north of the slides and extend below the slides in the eastern part of the area [2, 3, 5]. However, a geotechnical boring drilled over a well defined BSR area [10], has shown no evidence for gas or gas hydrates.

The HYDRATECH cruise on N/O Le Suroît (June-July 2002) aimed at a high resolution survey of a limited area of the Storegga slides. Data collected during the cruise include bathymetric and reflectivity EM300 maps (fig. 1), chirp echosounder records (fig. 3c) and high resolution (HR) 2D (fig. 2) and 3D (fig. 4) seismic data [13]. The study area (fig. 1) (about $3200 \mathrm{~km}^{2}$ ) extends from the upper slope at the northern boundary of the slides, to within the slides, in over $2 \mathrm{~km}$ water depth. It crosses slide scars and zones of potential present-day mechanical instability on the northern flank of the slides. It covers fields of pockmarks and various intra-sedimentary structures interpreted as fluid escape structures.

Seismic data interpretation confirms that, as proposed in Bouriak et al. [2] and Bünz et al. [5], the existence, location and amplitude of the BSR is controlled by the sediments properties. The main controlling factor would be the sediments ability to favour circulation and trapping of free gas below the base of the gas hydrate stability zone (BGHS). The BSR could then mainly be due to free gas trapped below the BGHS, without any indication of high gas hydrate concentrations above, in agreement with the low gas hydrate concentration inferred from refraction seismic data analysis [5].

High resolution seismic lines point to intense small-scale fracturing and diffuse degassing through the near seafloor sediments in the slides (fig. 2). In contrast, the slope area immediately north of the edge of the Storegga slides is nearly void of any seismic evidence 
pointing to seafloor degassing structures. Further upslope, fields of numerous gas chimneytype structures are observed starting at a distance of 10-20 km from the slides, in water depth of less than $1 \mathrm{~km}$ (fig. 1). High-resolution images obtained with a 2-5 kHz chirp profiler illustrate various types of these structures (fig. 3c). Not all of the chimneys are observed to reach the seafloor. When they do, the chimneys are often associated with seafloor depressions or pockmarks. Bathymetry and acoustic seafloor imagery data (fig. 3a and 3b) show that these pockmarks can be up to $500 \mathrm{~m}$ large in diameter, and are usually associated with a higher seabed reflectivity. They are shallow, rarely exceeding a few meters in depth. Pull-downs are commonly observed in and below the chimneys, which suggests the presence of gas. In several cases, surrounding reflectors are bent upwards near the chimneys, which may result from sediment deformation or an increase in velocity in the surrounding sediments. Most of the chimney-like structures are rooted below the BSR. On seismic and chirp profiles, amplitude anomalies are observed in the undisturbed sediments above the chimneys that do not reach the seafloor. These anomalies affect the sediments up to the seafloor and suggest recent fluid expulsion activity of these chimneys.

Bathymetric data (fig. 1) point to preferential directions in the slide scars and suggest a tectonic control of the sliding process. On the 3D seismic block (fig. 4), two shallow disturbed areas were identified located at about $150 \mathrm{~m}$ below sea bed. Those slides affect thin (about $15 \mathrm{~m}$ for the upper slide) layers in the Naust sedimentary formation, extend over a large part of the study area and are sealed by posterior sedimentation which implies early sliding of the sediments after deposition. The upper slide affects layers dated approximately 120000 years bp. There is no clear link between these slides and gas hydrates occurrence in the sediments: gas hydrate dissociation that could have had an impact on the strength of the sediments is expected to take place preferentially at the BGHS. However, the slides are nowadays still located well above the BGHS. The sliding layers are identified as glide planes for the -8200 years main Storegga slide event [7] which means that the instability of these layers could have lasted since the last 120000 years. In places where these layers are undisturbed the present day stability is thus still in question. 


\section{1/ Introduction}

La zone de Storegga est caractérisée par l'existence d'hydrates de gaz dans les sédiments de la pente continentale et la présence reconnue de grands glissements. C'est donc une région privilégiée pour l'étude des interactions entre présence de fluides et d'hydrates de gaz et instabilités de pente. La déstabilisation des hydrates de gaz, lors du réchauffement qui a suivi la dernière glaciation, est une hypothèse avancée pour expliquer le déclenchement des glissements de Storegga. Réciproquement, se pose la question de la quantité de méthane libérée vers l'atmosphère lors du rétrécissement de la zone de stabilité des hydrates suite aux grands glissements de Storegga. Pour apporter des éléments de réponse à ces questions, la campagne HYDRATECH se proposait d'établir une image sédimentaire précise, à l'aide d'outils géophysiques à haute définition, d'un secteur du flanc nord des glissements de Storegga. Il s'agissait de mettre en lumière la distribution du gaz et des hydrates de gaz dans les sédiments, les différentes structures d'échappement de fluides, les déformations intra sédimentaires, ainsi que les liens éventuels entre ces différents phénomènes. A travers la présentation d'extraits de données acquises sur la zone, cette contribution illustre l'intérêt de combiner différents outils géophysiques dont les données se complètent pour rendre compte des phénomènes physiques différents qui affectent la pente continentale.

\section{2/ La zone de Storegga}

\section{1/ Contexte géologique}

La fin de la formation de la marge de Norvège lors de l'ouverture de l'Atlantique Nord coïncide avec la transition Paléocène-Eocène il y a environ 55 Ma [6]. Du Miocène au Pliocène, des sédiments fins hémipélagiques se déposent sur la marge : les séries de la formation Kai. Cette formation est affectée par un système de failles polygonales dont l'origine est liée à des expulsions de fluides [1]. Du Pliocène au Pleistocène, la marge subit plusieurs épisodes glaciaires qui déposent sur le plateau un prisme épais de sédiments : la formation Naust [14]. De 2.5 à 1.3 Ma, la progradation de la plateforme remplit la dépression de Storegga. Lors des 1.3 derniers millions d'années, la sédimentation est conditionnée par l'alternance de cycles glaciaires et interglaciaires [11], avec une activité maximale vers 0.60.5 Ma lorsque la calotte glaciaire Fenno-Scandienne couvre la zone à répétition.

\section{2/Les glissements de Storegga}

Les glissements de Storegga qui affectent les séries actuelles ont été cartographiés sur plus de $800 \mathrm{~km}$, depuis la cicatrice en tête des glissements sur la partie haute de la pente continentale, vers $400 \mathrm{~m}$ de profondeur, jusqu'à la limite des sédiments déplacés dans la plaine abyssale du bassin norvégien, à des profondeurs de $3600 \mathrm{~m}$ [4]. A ce jour, huit glissements récents qui auraient fonctionné de façon rétrogressive et sur une courte durée (quelques heures) ont été identifiés [8]. Alors que le glissement principal de Storegga a été daté de 7250-7300 ans C14 (8150-8200 bp), des datations récentes sur le flanc nord font état de glissements à 5000 +/300 ans C14 et 2200-2500 ans C14, ce qui pose la question du risque actuel d'instabilité de cette zone [8].

\section{3/ Hydrates de gaz et BSR}

Les hydrates de gaz sont composés de molécules d'eau organisées en cages, qui piègent des molécules de gaz [12]. Leur formation est en particulier contrôlée par des conditions thermodynamiques particulières qui sont atteintes sur la plupart des marges continentales à des profondeurs d'eau comprises entre 500 et $1000 \mathrm{~m}$ : fortes pressions, faibles températures. L'observation d'un Bottom Simulating Reflector (BSR) est aujourd'hui encore l'indice géophysique principal pour conclure à la présence d'hydrates de gaz. Ces réflecteurs sismiques, à la limite inférieure de la zone de stabilité des hydrates de gaz [9], suivent les ondulations topographiques du fond de mer, en recoupant les réflecteurs sédimentaires stratigraphiques. Ils sont caractérisés par une polarité négative, qui exprime, suivant les 
résultats de la plupart des études entreprises, la présence de gaz libre sous les sédiments contenant les hydrates.

Sur la zone de Storegga, des BSRs ont été observés sur des profils sismiques au nord des glissements. Ils s'étendent à l'Est sous les glissements [2, 3, 5]. Un forage géotechnique a été réalisé dans une zone à BSR [10], mais ni hydrates, ni gaz libre n’ont été échantillonnés.

\section{3/ La campagne Hydratech : Outils utilisés et données}

Trois outils géophysiques principaux ont été utilisés. Ils ont permis d'obtenir des données complémentaires de surface et de sub-surface sur la zone.

\section{1/ Bathymétrie / Imagerie}

Le sondeur multifaisceaux EM300 du N/O Le Suroît a permis de relever une carte bathymétrique (fig. 1) et une carte de réflectivité acoustique du fond sur l'ensemble de la zone d'étude (40x60 km).

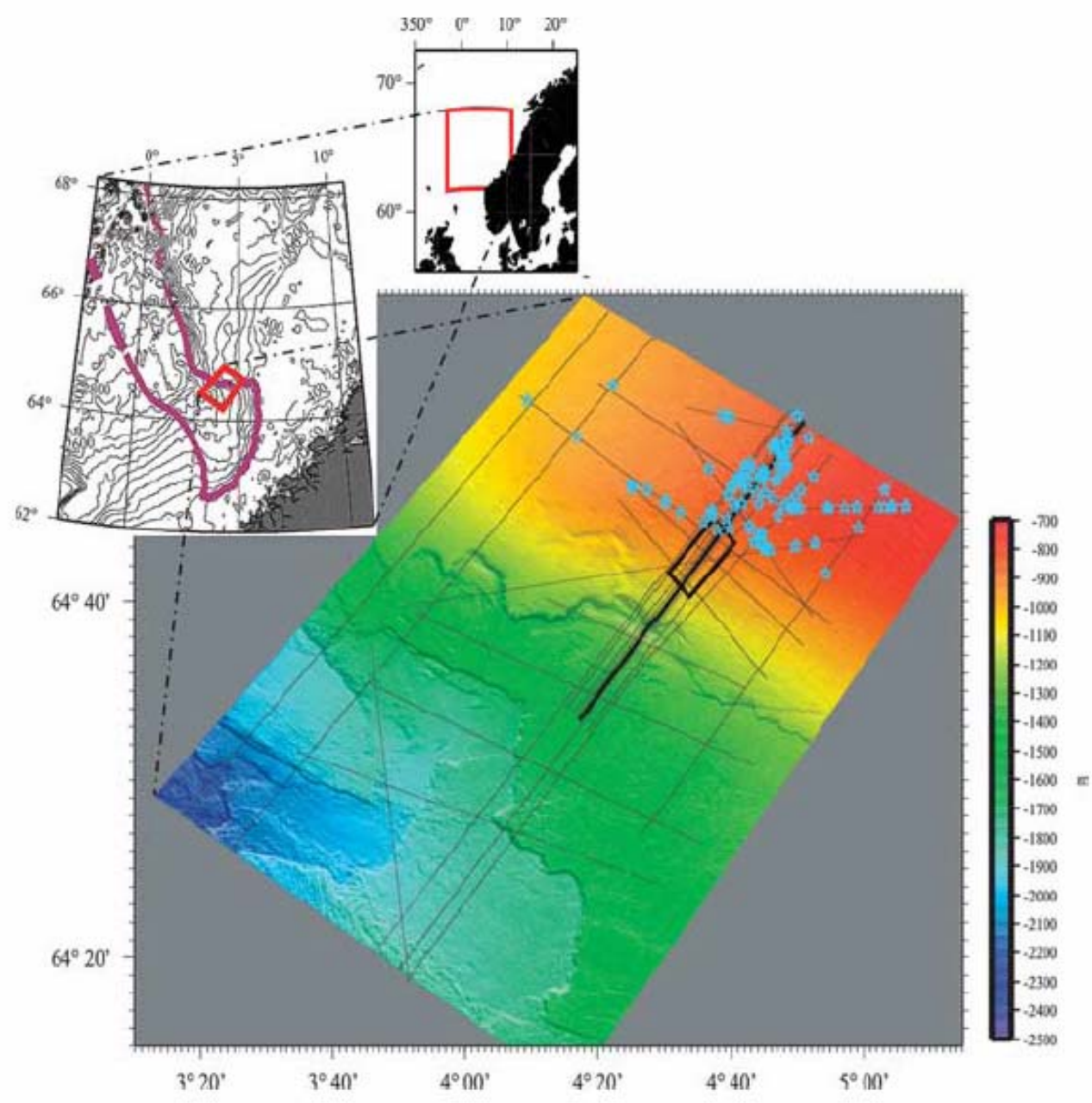

Figure 1 : Situation géographique et carte bathymétrique de la zone d'étude. En traits fins, position des profils sismiques haute résolution 2D (en trait gras, la ligne présentée figure 2). Le rectangle en trait gras donne la position du bloc de données sismiques 3D. Les étoiles signalent les cheminées de sorties de fluides détectées sur les profils sismiques.

Figure 1: Location and Bathymetric map of the study area. Thin lines: 2D high resolution seismic lines (thick line presented on figure 2). Bold line box: 3D data block. Stars: location of fluid escapes chimneys detected on the seismic lines. 


\section{2/ Imagerie sismique haute résolution}

Un outil de sismique haute résolution a été opéré pour : d’une part acquérir des profils régionaux (sismique $2 \mathrm{D}$ conventionnelle) et d'autre part , pour la première fois dans le milieu universitaire, acquérir des données de sismique 3D couvrant une surface limitée.

\subsection{1/ Sismique Haute Résolution 2D}

Une série de 24 profils haute résolution, enregistrés sur une flûte numérique de $750 \mathrm{~m}$ (120 traces de $6.25 \mathrm{~m}$ ) immergée à $3 \mathrm{~m}$, a été acquise. La source était composée de 2 canons à air mini-GI guns immergés à $1.5 \mathrm{~m}$, qui permettent d'émettre dans une gamme de fréquences de 20 à $300 \mathrm{~Hz}$. Après application d'un filtre passe bande et correction de l'atténuation géométrique, les données ont été sommées et migrées en profondeur en prenant en compte la navigation réelle des sources et des récepteurs (fig 2).

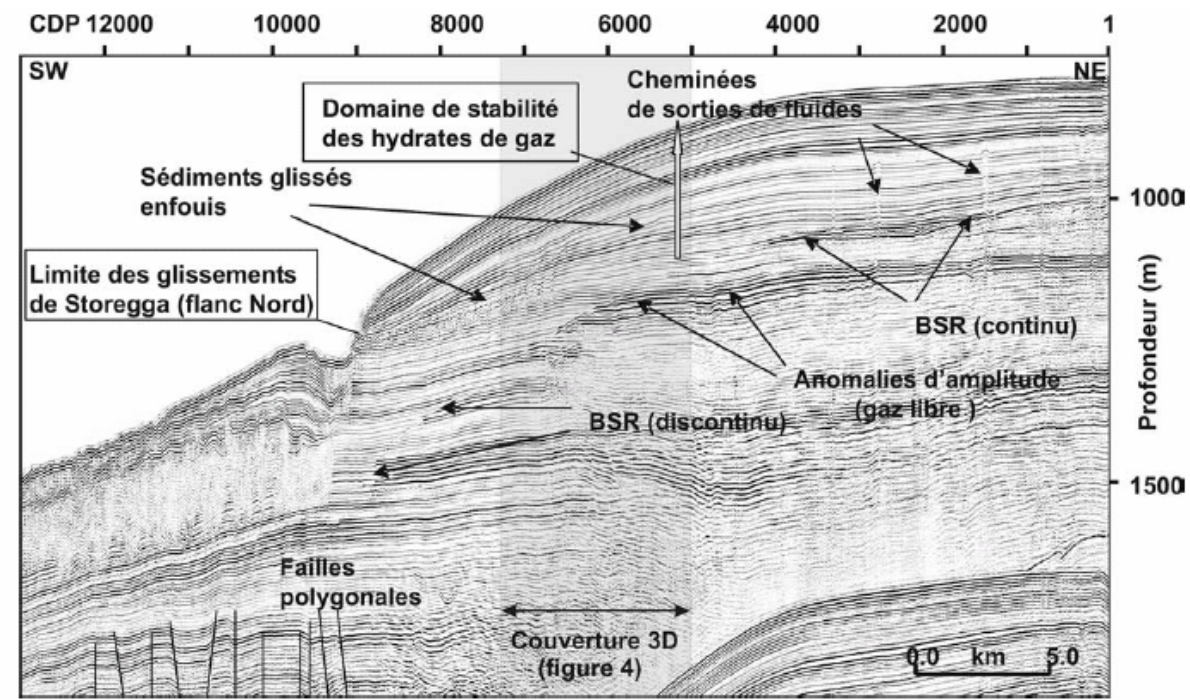

Figure 2 : Profil HTK2D - 05 migré en profondeur. L'axe des abscisses est repéré par le numéro de CDP (Common Depth Point) ou Point Milieu Commun. Noter que le BSR (Bottom Simulating Reflector) est matérialisé par un réflecteur continu au NE et par la terminaison d'anomalies d'amplitudes au SW du CDP 9000. La zone grisée couverte par le bloc sismique 3D est présentée en figure 4.

Figure 2: Line HTK2D - 05 depth migrated. Horizontal axis scale: CDP (common depth points). Note that the BSR (Bottom Simulating Reflector) is marked by a continuous reflector at the NE end of the line and by the end of amplitude anomalies SW of CDP 9000. The grey shaded area corresponds to the 3D data displayed on figure4.

\subsection{2/ Sismique Haute Résolution 3D (HR 3D)}

Un système d'acquisition original développé à l’Ifremer [13] a été mis en œuvre pour la première fois dans le cadre d’une campagne scientifique. Le dispositif comprend deux flûtes de 48 traces chacune (intertrace de $6.25 \mathrm{~m}$ ) et deux sources fonctionnant alternativement. Quatre lignes “point milieu” sont éclairées par ligne naviguée, d’où un gain de temps d'acquisition considérable par rapport à un dispositif conventionnel composé d'une source et d'une flûte. L'intertir est d'environ $6.25 \mathrm{~m}$. Les lignes naviguées sont espacées de $25 \mathrm{~m}$, ce qui a permis de couvrir une zone de $3.5 \times 7 \mathrm{~km}$ en 12 jours environ. Le bloc de données 3D obtenu a une résolution horizontale théorique de 6.25x3.125 m (dimensions du bin) et une résolution verticale de l'ordre du mètre. La qualité de l'image 3D finale dépend en grande partie du traitement soigné des données de navigation. La phase de prétraitement des collections points de tir comprend en particulier filtrage fréquentiel et corrections statiques. L'ensemble des données est ensuite migré en profondeur (migration avant sommation de type Kirchoff). Le bloc migré présenté a été obtenu en utilisant un gradient de vitesse dans les 
sédiments estimé à l'aide d'analyses de vitesse sur les lignes HR2D régionales. Les données sismiques ont été interprétées à l’aide du logiciel Sismage (Total).

\section{3/ Sondeur Chirp}

Le Suroît est équipé d’un sondeur de sédiments de coque émettant un signal modulé en fréquence sur une bande de fréquences assez large $(2.5-4.5 \mathrm{kHz})$. Il a été utilisé en mode chirp avec un signal d'une durée de $10 \mathrm{~ms}$, à $25 \%$ de la puissance maximale du sondeur, pendant douze heures par jour sur toute la durée de la campagne (24 jours sur zone). Les données sont de bonne qualité, avec une pénétration moyenne de 150 à 200 ms temps double (fig. 3).
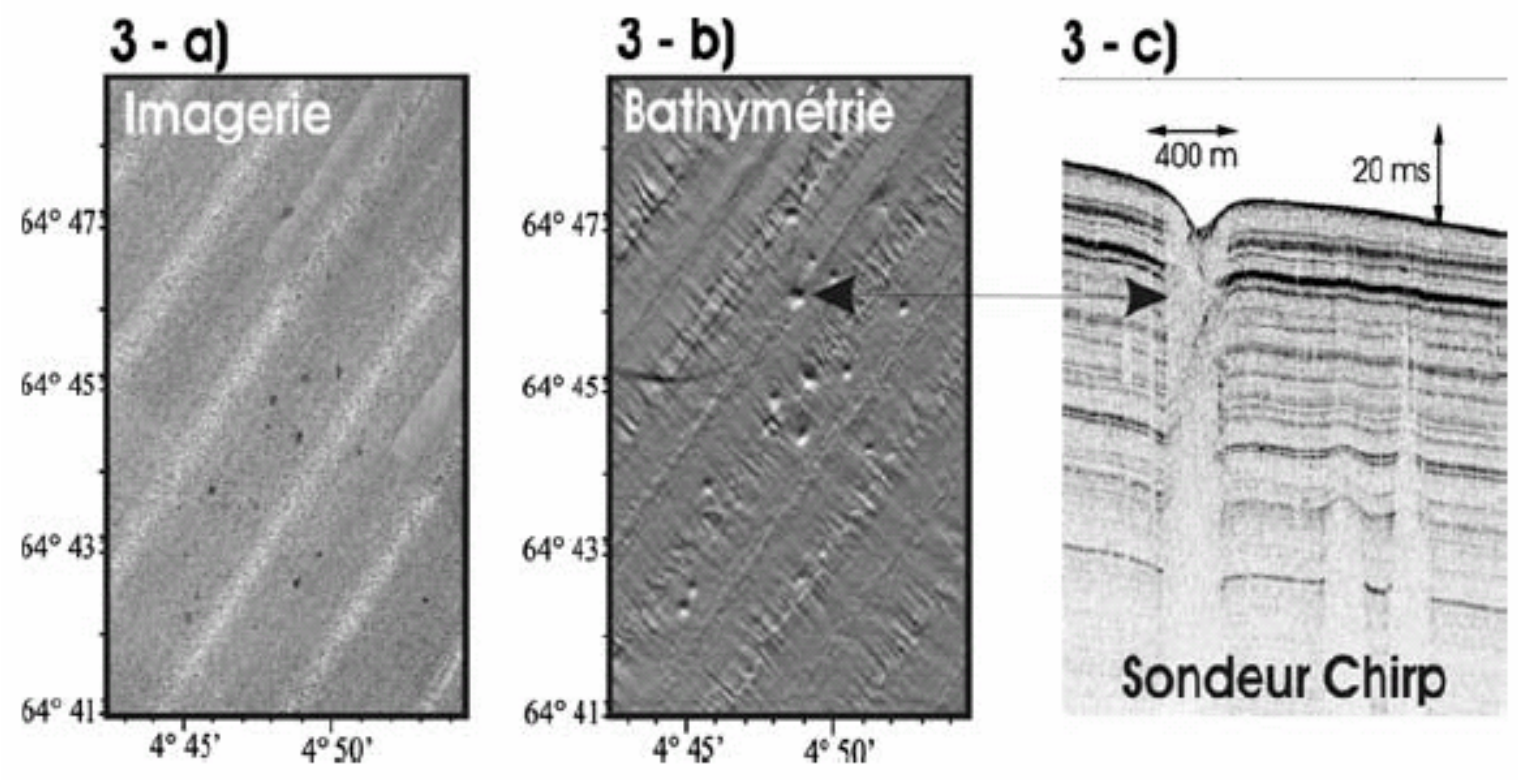

Figure 3 : Expression des sorties de fluides, sur un zoom des cartes de bathymétrie (3-a) et d’imagerie (3-b), et sur un enregistrement du sondeur de sédiments (3-c).

Figure 3: Fluid escapes on a zoom of the bathymetric (3-a) and imagery (3-b) maps and on a record of the chirp sediment profiler (3-c).

\section{4/ Données et interprétations préliminaires}

\section{1/ Hydrates de gaz et Bottom Simulating Reflector (BSR)}

Le profil htk2D01-1 présenté en figure 2, illustre les caractéristiques principales du BSR sur la zone d'étude. On peut noter en particulier les propriétés variables de ce réflecteur. Il est continu sur le haut de la pente entre les points milieu commun (CDP) 1000 à 4000. A cet endroit, il est matérialisé par un réflecteur de polarité inverse par rapport au fond de l'eau et presque coïncident avec la stratigraphie. Le BSR disparaît alors (CDP 4500 et 6500) en tant que réflecteur. La base du domaine de stabilité des hydrates de gaz (notée dans la suite BHSZ pour Bottom of Hydrate Stability Zone) est définie par la terminaison d'anomalies d'amplitude. Ensuite, et jusqu'à environ $2 \mathrm{~km}$ au SW de la limite nord des glissements de Storegga, la BHSZ est alternativement marquée par des segments de réflecteur ou des terminaisons d'anomalies d'amplitude (CDP 6500 à 9000). Enfin, sous la zone affectée par les glissements (CDP 9000 à 13000), anomalies d'amplitude et BSR ne sont pas visibles. Il faut noter que la profondeur du BSR en limite des glissements coïncide avec la base des niveaux sédimentaires affectés par les glissements. Une carte de la répartition du BSR sur la zone d'étude 3D a été réalisée à partir des données de la campagne. Les zones où le BSR apparaît en tant que réflecteur continu à la résolution de la sismique utilisée et celles où il se 
matérialise par la terminaison d'anomalies d'amplitude ont été cartographiées. Cette carte confirme le caractère discontinu du BSR. En particulier, l'amplitude relative du BSR dépend de la strate intersectée par celui-ci. Ces variations d'amplitude pourraient être dues à des quantités variables de gaz piégées sous le BSR, en fonction de la porosité et de la perméabilité des séries sédimentaires recoupées par le BSR. On note aussi, dans le bas de la zone 3D un changement de l'amplitude relative du BSR lié à la présence d'une faille. En première analyse, la présence et l'amplitude du BSR sont donc contrôlées par la nature des séries sous jacentes ainsi que par l'existence de failles intra-sédimentaires. Il semble que ce soit principalement la capacité des séries sédimentaires à piéger le gaz au niveau de la BHSZ qui contrôle ces amplitudes. Aucune anomalie d'amplitude qui pourrait être reliée à la présence d’hydrate dans les séries au dessus du BSR n’est observée.

\section{2/ Sorties de fluides}

Les cartes bathymétriques (fig. 1 et 3b) et d'imagerie (fig. 3a) mettent en évidence de nombreuses structures sub-circulaires d'un diamètre moyen de $300 \mathrm{~m}$ environ, et qui affectent le fond de l'eau en haut de pente de la zone d'étude. La plupart de ces structures sont des dépressions (pockmarks) sur la bathymétrie et correspondent à des zones de forte réflectivité sur l'imagerie. On peut observer des structures en dômes, mais celles-ci sont plus rares. Ces structures sont corrélées sur les profils de sondeur de sédiment et sur les profils sismiques (fig. 2, fig. 3c, fig. 4) à des zones verticales (cheminées) le long desquelles les réflecteurs semblent interrompus. Certaines de ces cheminées atteignent le fond de l'eau, d'autres semblent être scellées. Une carte de la répartition des cheminées réalisée à partir des données sismiques (fig. 1) montre qu'elles se développent au dessus d'une profondeur d'environ 950-900 m à l'est et de 1050-1000 m à l'ouest (fig. 1). A la périphérie de ces cheminées, les réflecteurs sont rebroussés vers le haut, ou vers le bas, ou encore très peu affectés (fig. 3c). Sur les profils sismiques (fig. 2, fig. 4), les cheminées peuvent être suivies en profondeur jusqu'au BSR. Il est difficile de préciser si elles affectent effectivement les réflecteurs plus profonds ou si elles se terminent au niveau des anomalies d'amplitude dans les horizons sous le BSR. Une analyse détaillée des profils sismiques et de sondeur de sédiments montre des anomalies d'amplitude qui affectent les séries non déformées à la verticale des cheminées scellées, et ce, jusqu'au fond de l'eau. Ces anomalies suggèrent une activité récente ou actuelle d'expulsion de fluides à travers ces cheminées. Particulièrement bien visibles sur les profils de sondeur de sédiment, mais aussi sur la sismique, des failles normales de faible rejeu (quelques $\mathrm{m}$ ) limitent de petites zones effondrées. Le bloc sismique 3D permet de montrer que ces structures qui pourraient être assimilées à de petits grabens d'extension sur une ligne 2D ont en réalité une forme circulaire et pourraient représenter un stade peu évolué de ces cheminées. 


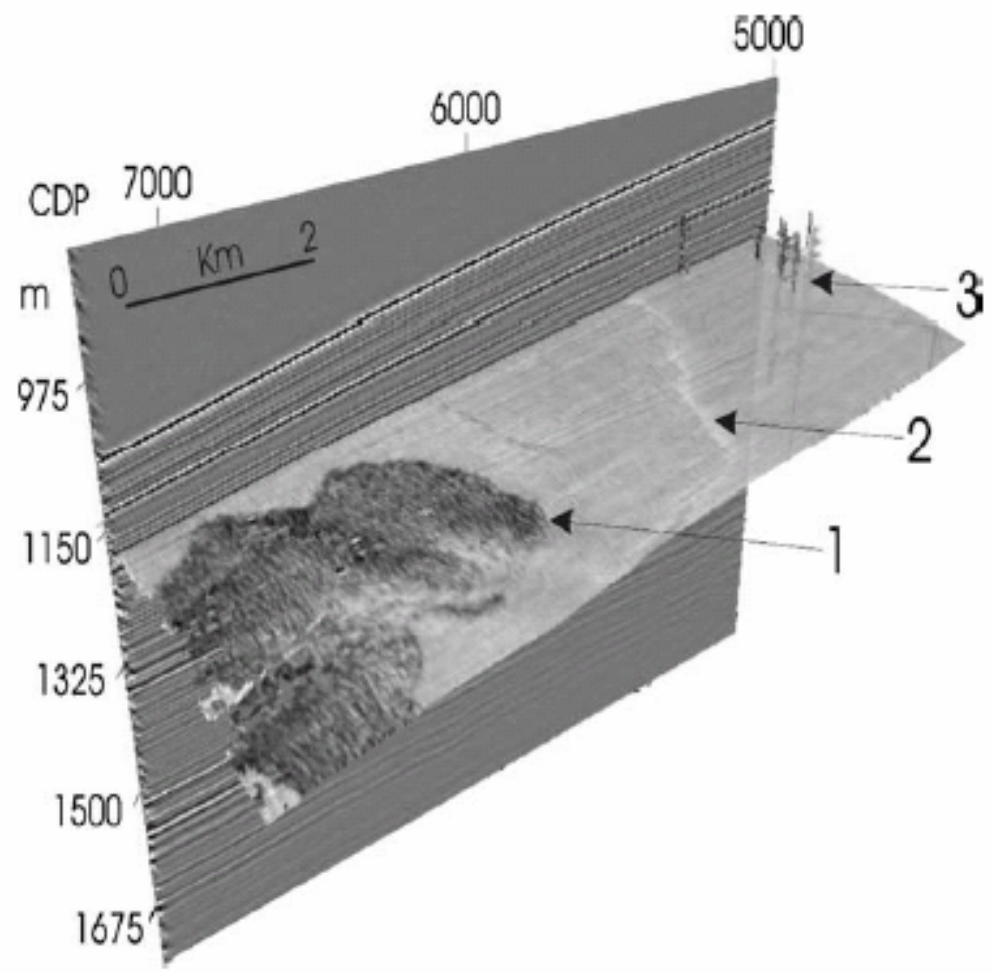

Figure 4 : Image 3D d'un paléo glissement : carte de l'amplitude relative de l'horizon glissé extraite du bloc de données sismiques 3D. 1 = limite haute des sédiments fortement glissés, 2 = limite haute des sédiments peu déplacés, 3 = cheminées de sorties de fluides.

Figure 4: 3D picture of a paleo slide: amplitude map of the disturbed horizon extracted from the 3D data block. 1 = upslope limit of the highly perturbed sediments, 2 = upslope limit of the slightly disturbed sediments, $3=$ fluid escape chimneys.

\section{3/ Glissements}

Le niveau de détail des cartes bathymétrique (fig. 1) et d'imagerie apporte des informations essentielles : il permet de définir la limite des sédiments perturbés par les glissements récents ou encore met en évidence à l'intérieur de la zone glissée, plusieurs plans de glissement situés à des niveaux différents, et dont les limites quasi linéaires semblent orientées dans des directions préférentielles (fig. 1). Il est possible aussi d'identifier en bordure de glissement des zones perturbées mais dont le déplacement horizontal est très faible. Sur les profils sismiques 2D, ces zones correspondent à des séries litées en surface, au dessus d'un faciès acoustiquement sourd avec quelques réflexions discontinues à la base.

Pour l'identification des instabilités sédimentaires anciennes, dans les séries de la zone investiguée, c'est l'analyse du bloc de données sismiques 3D qui se révèle particulièrement intéressante. La figure 4 représente la carte en 3D d'un horizon situé à environ $150 \mathrm{~m}$ sous le fond. Sur un profil 2D (fig. 2), cet horizon est continu au NE, puis perturbé à partir du CDP 5500 et enfin très discontinu après le CDP 6500. En carte, on montre immédiatement que cet horizon est affecté par un glissement dont l'ampleur du déplacement horizontal s'accroît à partir du CDP 6500 (fig. 2 et 4). La partie supérieure du glissement (CDP 5500-6500) présente un déplacement faible ; la partie inférieure a plus largement mobilisé les sédiments, sur une distance plus importante. Ces deux phases de glissement semblent toutefois scellées par les séries sédimentaires immédiatement postérieures, ce qui implique un déclenchement précoce de ces instabilités. Un second niveau glissé avec un comportement similaire au 
précédent peut être identifié à une profondeur de quelques dizaines de $\mathrm{m}$ sous le premier glissement. Les séries affectées par ces deux glissements appartiennent à la formation Naust. Plus précisément, le glissement supérieur affecte des séries de la formation Naust, datées de 120000 ans BP [7].

\section{5/ Discussion}

L'analyse des données de la campagne Hydratech, en particulier celle des cartes d'amplitude issues du bloc 3D, confirme le contrôle de la position et de l'amplitude du BSR par la nature des séries sédimentaires qui recoupent la limite inférieure de la BGHS. Comme le proposent Bouriak et al. [2] et Bünz et al. [5], le facteur principal de contrôle du BSR est probablement la capacité de ces séries à permettre la circulation et le piégeage du gaz libre sous la BGHS. Si le BSR est principalement marqué par le toit de séries chargées en gaz libre, sa présence n'est donc pas synonyme de fortes teneurs en hydrates au dessus du BSR. L'absence de réflecteur susceptible de marquer le toit des hydrates ainsi que la faible teneur en hydrates (inférieure à quelques \% de la porosité) estimée à partir de données de sismique réfraction sur la zone par Bunz et al. [5] corroborent ce raisonnement.

L’analyse conjointe des données de bathymétrie, de sondeur de sédiment et de sismique HR permet d'identifier de nombreuses cheminées de sorties de fluides et de montrer que celles ci sont actives ou ont été actives très récemment car même si elles ne sont pas marquées par un relief sur le fond de l'eau, l'amplitude du fond de la mer sur les profils chirp et sismique est affectée à la verticale de ces cheminées. A partir des données acquises, on montre que les cheminées s'enracinent sous le BSR, mais il n’est pas possible de préciser si c'est au niveau des séries chargées en gaz immédiatement sous le BSR ou plus profondément. Leur répartition limitée au haut de pente pourrait être contrôlée par l'existence de surpressions liées à la présence, mise en évidence par les données sismiques, de gaz libre dans les séries sous le BSR.

Les deux glissements décrits dans le bloc sismique 3D se sont déclenchés peu après le dépôt des séries glissées comme le prouve le scellement de ces séries par les sédiments postérieurs. Ils sont caractérisés par une faible épaisseur (de l'ordre de $15 \mathrm{~m}$ en moyenne pour le glissement supérieur), et par une extension horizontale régionale. A partir des données disponibles, il n'existe pas de lien évident entre la présence de gaz ou d'hydrates de gaz et ces deux glissements superficiels observés sur le bloc 3D : les glissements mis en évidence sont éloignés de la BGHS et l'étaient d'autant plus lors de leurs mises en place. Or, c'est principalement au niveau de la BGHS que la déstabilisation des hydrates de gaz contenus dans les sédiments est susceptible de se produire et d'influencer la stabilité de ces derniers.

Les plans de glissement identifiés ont joué ultérieurement un rôle lors du glissement principal de Storegga [7]. L’instabilité de ces séries aurait donc perduré depuis leur dépôt daté de 120000 ans [7] jusqu'à -8200 ans, date de déclenchement du glissement de Storegga [8]. Sur son flanc nord, les zones où ces séries sont encore intactes pourraient alors continuer de présenter un risque d'instabilité important.

\section{6/ Conclusion}

La combinaison des différentes données géophysiques acquises au cours de la campagne Hydratech apporte des informations sur les structures détaillées et la répartition : des séries sédimentaires ; du BSR ; des sorties de fluides et des glissements sur la zone étudiée. Une première analyse des données permet: 1/ de confirmer le contrôle prépondérant de la position et de l'amplitude du BSR par les séries sédimentaires ; 2/ de montrer l'activité récente ou actuelle des sorties de fluides dans la zone d'étude ; 3/ de minimiser l'influence des hydrates de gaz dans le déclenchement des deux glissements superficiels identifiés sur le secteur étudié du flanc nord des glissements de Storegga; 4/ de mettre en évidence le 
caractère durablement instable de certaines séries de la formation Naust, et donc l'instabilité potentielle des zones où ces séries sont encore intactes sur le flanc nord des glissements de Storegga.

En revanche, la question de l'impact de la possible déstabilisation des hydrates de gaz dans le déclenchement des glissements principaux de Storegga n'est pas résolue. La poursuite de l'exploitation des données de la campagne Hydratech est toutefois prometteuse.

\section{Remerciements}

La campagne Hydratech a été financée par l'Ifremer et le projet européen HYDRATECH. Nous remercions le Commandant Thierry Alix, les officiers et l'équipage du N/0 Le Suroît pour leur collaboration. 


\section{Références}

[1] C. Berndt, S. Bünz, and J. Mienert, "Polygonal fault systems on the mid-Norwegian margin: A long term source for fluid flow" in P. Van Rensbergen, R. R. Hillis, A. J. Maltman and C. K. Morley (Eds), "Subsurface Sediment Mobilisation”, Special Publication Geological Society of London (2003) 283-290.

[2] S. Bouriak, A. Volkonskaia, V. Galaktinov. "Split” strata-bounded gas hydrate BSR below deposits of the Storegga Slide and at the southern edge of the Voring Plateau. Marine Geology 195 (2003) 301-318.

[3] S. Bouriak., M. Vanneste, A. Saoutkine, inferred gas hydrates and clay diapirs near the Storegga Slide on the southern edge of the Voring Plateau, offshore Norway. Marine Geology 163 (2000) 125-148.

[4] T. Bugge, S. Befring, R.H. Belderson, T. Eidvin, E. Jansen, A Giant 3-Stage submarine slide off Norway. Geo-Marine Letters 7(4) (1987) 191-198.

[5] S. Bünz, J. Mienert, C. Berndt, Geological controls on the Storegga gas-hydrate system of the mid-Norwegian continental margin. Earth and Planetary Science Letters 209 (2003) 291-307.

[6] L. Fidalgo Gonzales. L'évolution de l'Atlantique Nord: la question de la déformation intraplaque Thèse de Doctorat de l'Université de Bretagne Occidentale (2001).

[7] H. Haflidason, H.P. Sejrup, I.M. Berstad, A. Nygard, T. Richter, P. Bryn, R. Lien, K. Berg. A weak layer feature on the Northern Storegga Slide Escarpment. In Mienert and Weaver (Eds), European Margin Sediment Dynamics (2003) 45-53.

[8] H. Haflidason, W. Lekens, R. Lien, P. Bryn, H.P., Sejrup, The dating of the Storegga Slide: Precison and problems. $4^{\text {th }}$ Costa Workshop, Bologna, Italy, Feb. 10-13 (2003).

[9] K. A. Kvenvolden and L. A. Barnard, Gas hydrates of the Blake Outer Ridge, Site 533, Deep Sea Drilling Project Leg 76. In Sheridan, R.E., Gradstein, F.M., et al., Init. Repts. DSDP, 76: Washington (U.S.Govt. Printing Office), (1983) 353-365.

[10] J. Mienert \& P. Bryn : Gas Hydrate Drilling conducted on the European Margin. EOS, Transactions, American Geophysical Union, 78, 49 (1997) 567-571.

[11] D. Ottesen, L. Rise, K. Rokoengen \& J. Sættem. Glacial processes and large-scale morphology on the mid-Norwegian continental shelf. In: Martinsen, O. \& Dreyer, T. (Eds), Sedimentary environments offshore Norway - Palaeozoic to Recent, Norwegian Petroleum Society (2001).

[12] E.D.J. Sloan. Clathrate hydrates of natural gases, second edition, revised and expansed. Chemical Industries, 73. Marcel Dekker, Inc., New York (1998) 705 pp.

[13] Y. Thomas, B. Marsset, S. Didailler, J.-P. Regnault, S. Le Conte, D. Le Roux, P. Farcy, M. Magueur, P. Viollette, J. Herveou, J.-C. Guedes, B. Jegot, G. Gascon, C. Prud'homme, H. Nouzé, E. Thereau, I. Contrucci, J.-P. Foucher. Sismique marine Haute 
Résolution 3D : Un nouvel outil de reconnaissance à destination de la Communauté Scientifique. C. R. Geoscience 336 (2004) 579-585.

[14] T.O. Vorren, J.S. Laberg, F. Blaume, J.A. Dowdeswell, N.H. Kenyon, J. Mienert, J. Rumohr, F. Werner. The Norwegian-Greenlan sea continental margins: Morphology and late quaternary sedimentary processes and environment. Quaternary Science Reviews, Vol. 17 (1998) 273-302. 